\title{
A PARTICULAR PHOTODYNAMIC THERAPY FOR THE TREATMENT OF REFRACTORY SPONTANEOUS MAXILLARY ONJ: A CASE REPORT
}

Cinzia Casu, Martina Salvatorina Murgia ${ }^{1}$, Valentino Garau ${ }^{1}$, Lia Ester Argiolas, Germano Orrù

1 University of Cagliari

Funding: The author(s) received no specific funding for this work.

Potential competing interests: The author(s) declared that no potential competing interests exist.

\section{Abstract}

Drug-related osteonecrosis of the jaw (MRONJ) is a drug complication characterized by the progressive destruction and necrosis of the bone, in the absence of previous radiation treatment. In the present report, a 58-year-old cancer patient treated with zolendronic acid developed a spontaneous palatal osteonecrosis. Following repeated courses of antibiotic therapy, there was no improvement of the osteonecrosis. Subsequently, after several attempts, an integrum healing occurred with the use of an $810 \mathrm{~nm}$ diode laser at $3.5 \mathrm{~W}$ and $10 \%$ povidone iodine as photosensitizer, with the addition of $0.330 \mathrm{~g}$ of acetylsalicylic acid. and $0.200 \mathrm{~g}$ of vitamin $\mathrm{C}$ as a final rinse. Therefore, the aim of our work is to illustrate the resolution of a spontaneous maxillary ONJ with this particular PDT.

\section{Background:}

Drug-related osteonecrosis of the jaw (MRONJ) is a potentially serious and debilitating drug-related complication. It consists in the progressive bone destruction in the maxillofacial area of patients exposed to treatment with drugs associated with the risk of $\mathrm{ONJ}$, in the absence of previous radiation therapy. The treatment involves the application of well-defined therapeutic ministerial protocols, however, there are numerous cases of relapse and incomplete remission of MRONJ. In the literature there are many alternative therapeutic options for the management and prevention of MRONJ, in particular, photodynamic therapy (PTD) is increasingly used with very positive outcomes ${ }^{[1-5]}$. Therefore, the aim of our work is to illustrate the resolution of a spontaneous maxillary ONJ with a particular PDT.

Materials and Methods: 


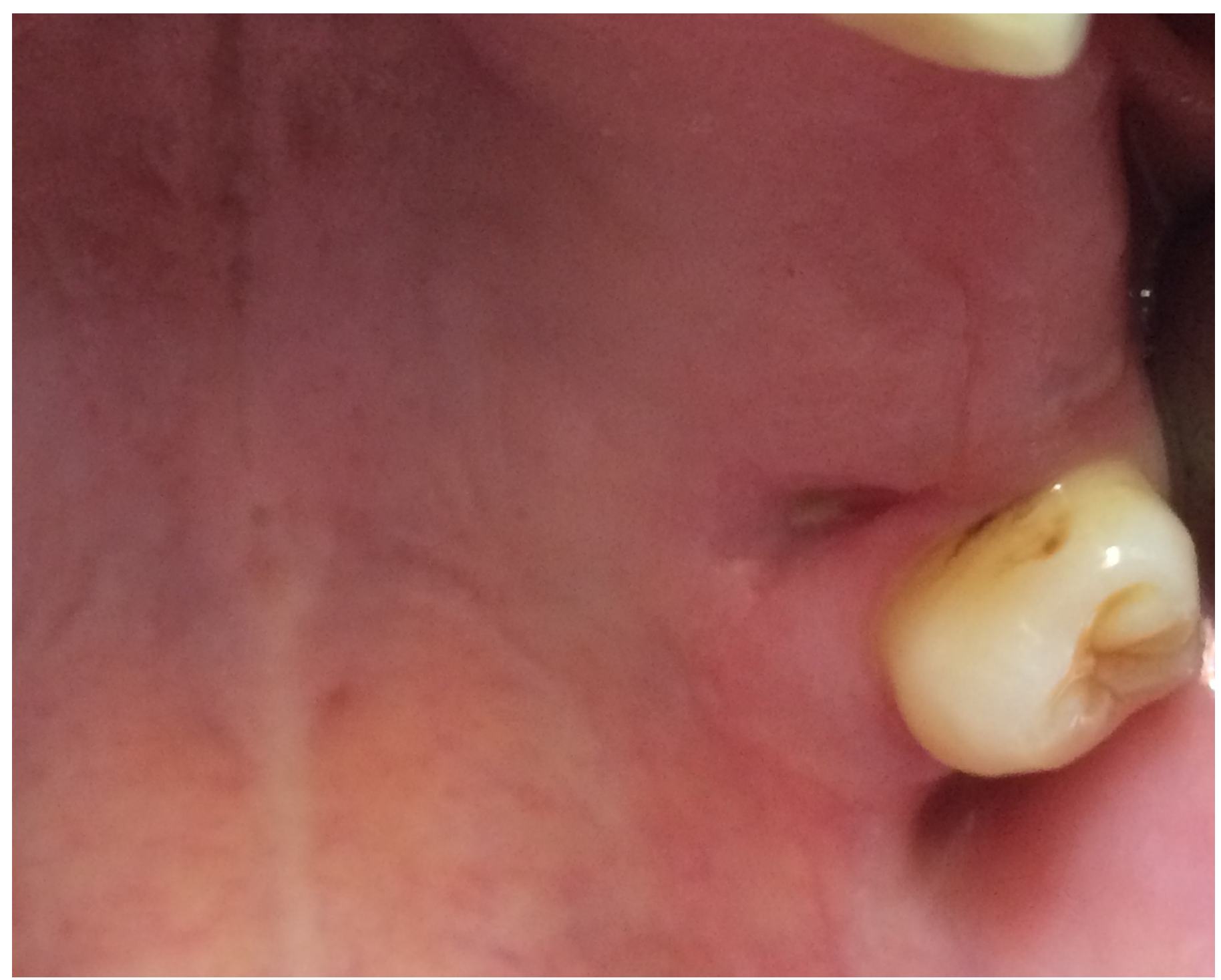

Fig. 1. MRONJ during the first visit. 


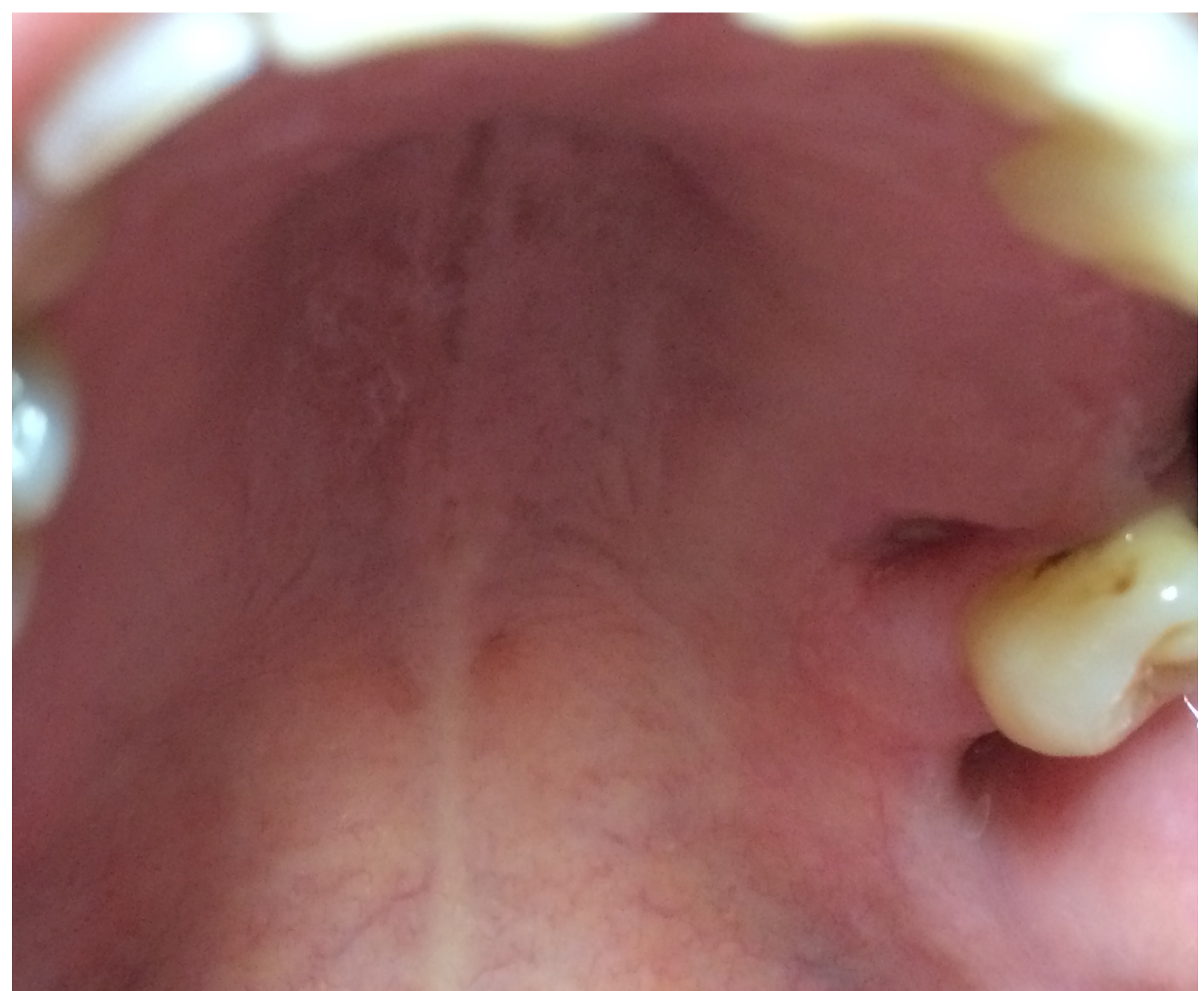

Fig. 2 MRONJ after antibiotic therapy and topical application of chlorhexidine gel.

A 58-years-old woman came to our attention for an intraoral lesion. The patient's medical history reported previous surgically treated breast cancer associated with intravenous administration of zoledronic acid. An intraoral examination revealed necrotic bone in the in the hemipalatal area of $1,5 \times 1,5 \mathrm{~cm}$ (Fig. 1). In the initial stages, several courses of antibiotic therapy based on amoxicillin + clavulanic acid (cp $3 \mathrm{~g} / \mathrm{day}$ ) and metronidazole ( $\mathrm{cp} 1.5 \mathrm{~g} /$ day) were performed for a duration of 14 days. In addition, topical application of chlorhexidine gel was prescribed. However, palatal bone exposure showed no improvement (Fig. 2). It was therefore decided, in agreement with the patient, to perform a PDT with diode light at $630 \mathrm{~nm}$ and 7 Watts of power (Fotosan, CMS Dental, Copenhagen, Denmark), combined with 1\% toluidine blue as photosensitizer. Again, there was no lesion's improvement. 


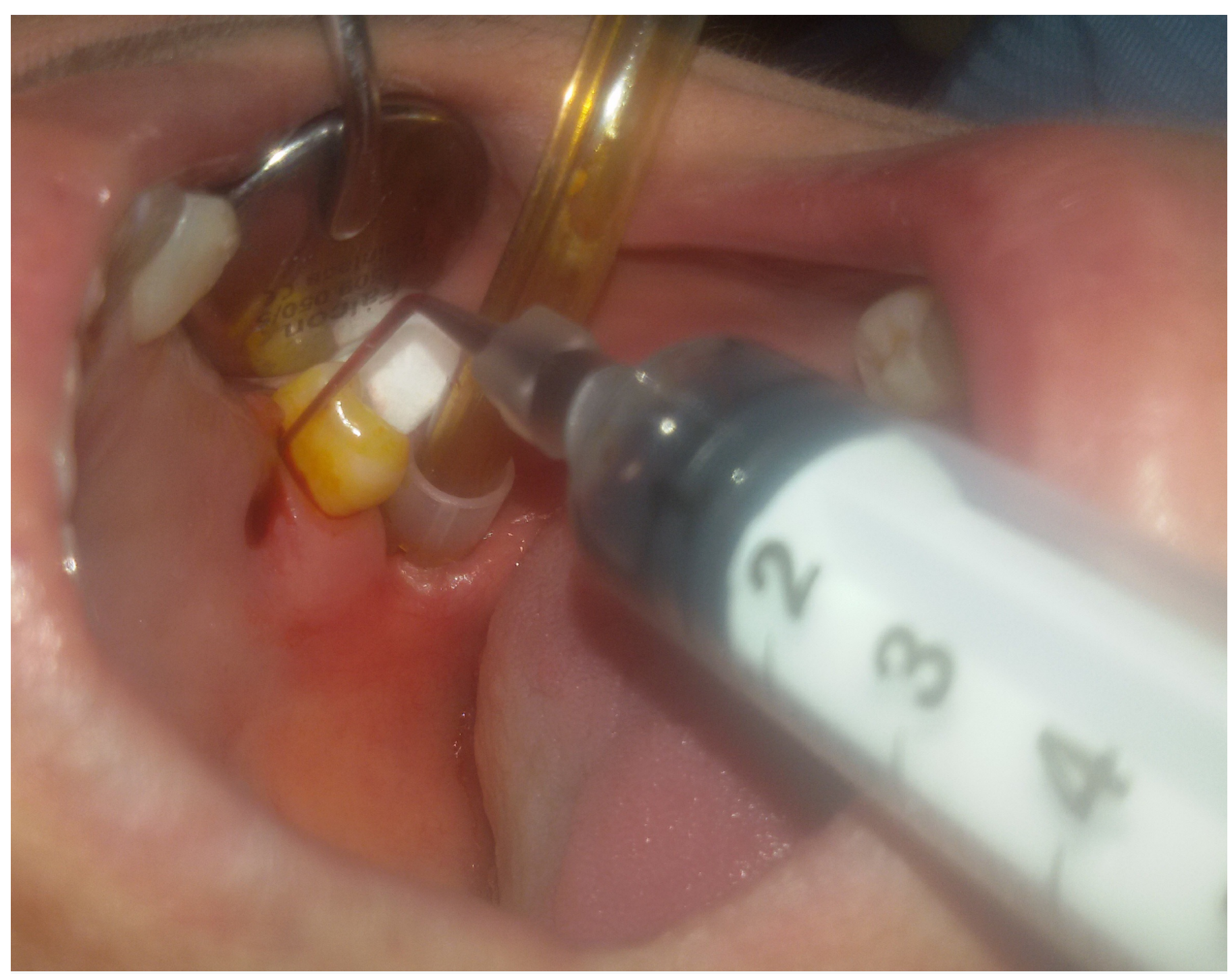

Fig. 3 Application of the povidone iodine as photosensitizer. 


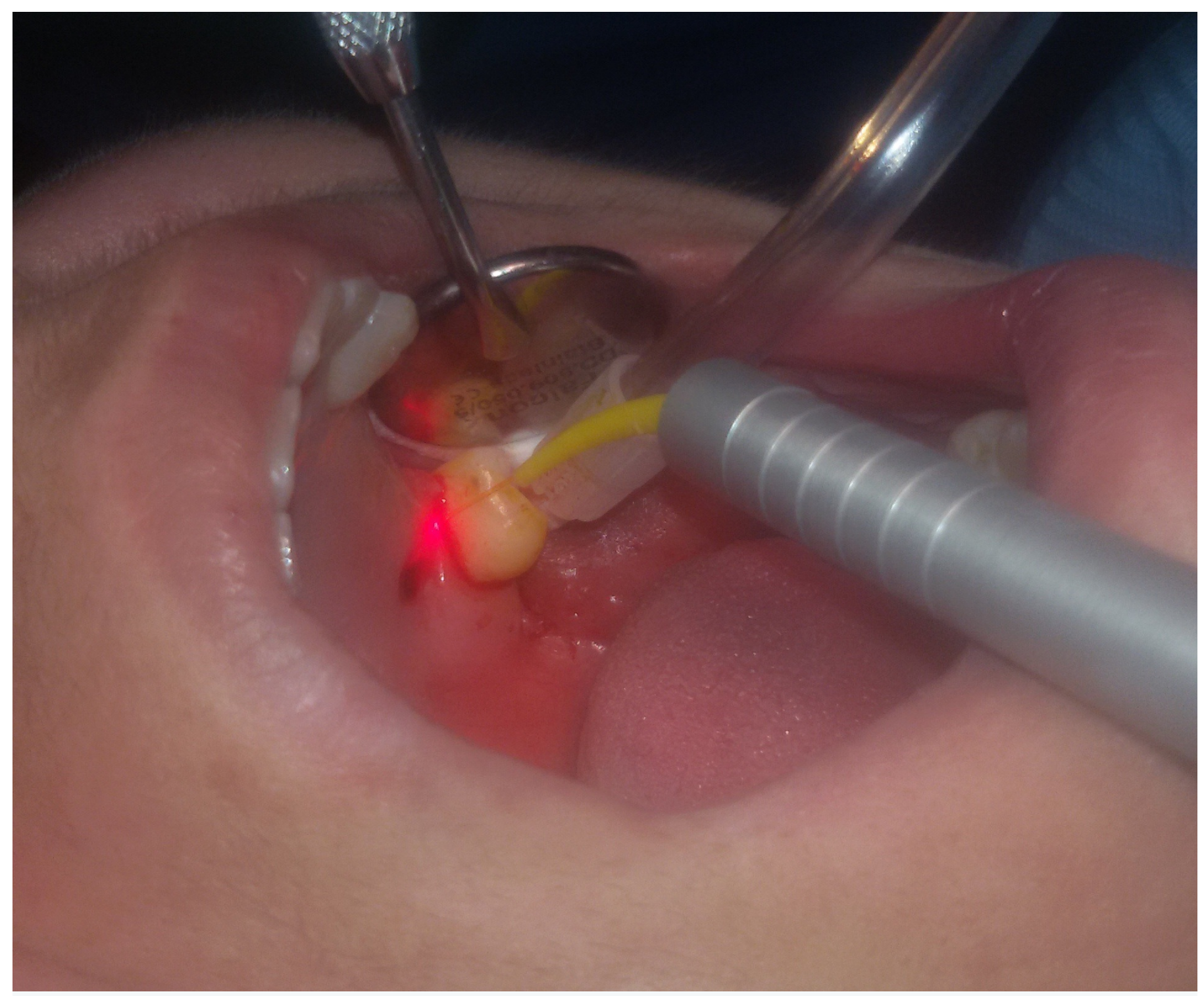

Fig. 4. Activation of the photosensitizer with $810 \mathrm{~nm}$ diode laser.

Finally, a PDT was performed with an $810 \mathrm{~nm}$ diode laser at $3.5 \mathrm{~W}$ and 10\% povidone iodine as photosensitizer (Fig. 3, 4), with the addition of $0.330 \mathrm{~g}$ of acetylsalicylic acid and $0.200 \mathrm{~g}$ of vitamin $\mathrm{C}$ dissolved in $200 \mathrm{ml}$ of water as a final rinse. The tip of the laser device had a 320 um diameter and was held $1 \mathrm{~mm}$ away from the osteonecrosis surface in continuous mode. As suggested by the company, the irradiation time was 60 seconds for each cycle. 5 cycles were performed with circular and continuous movements over the entire lesion. 


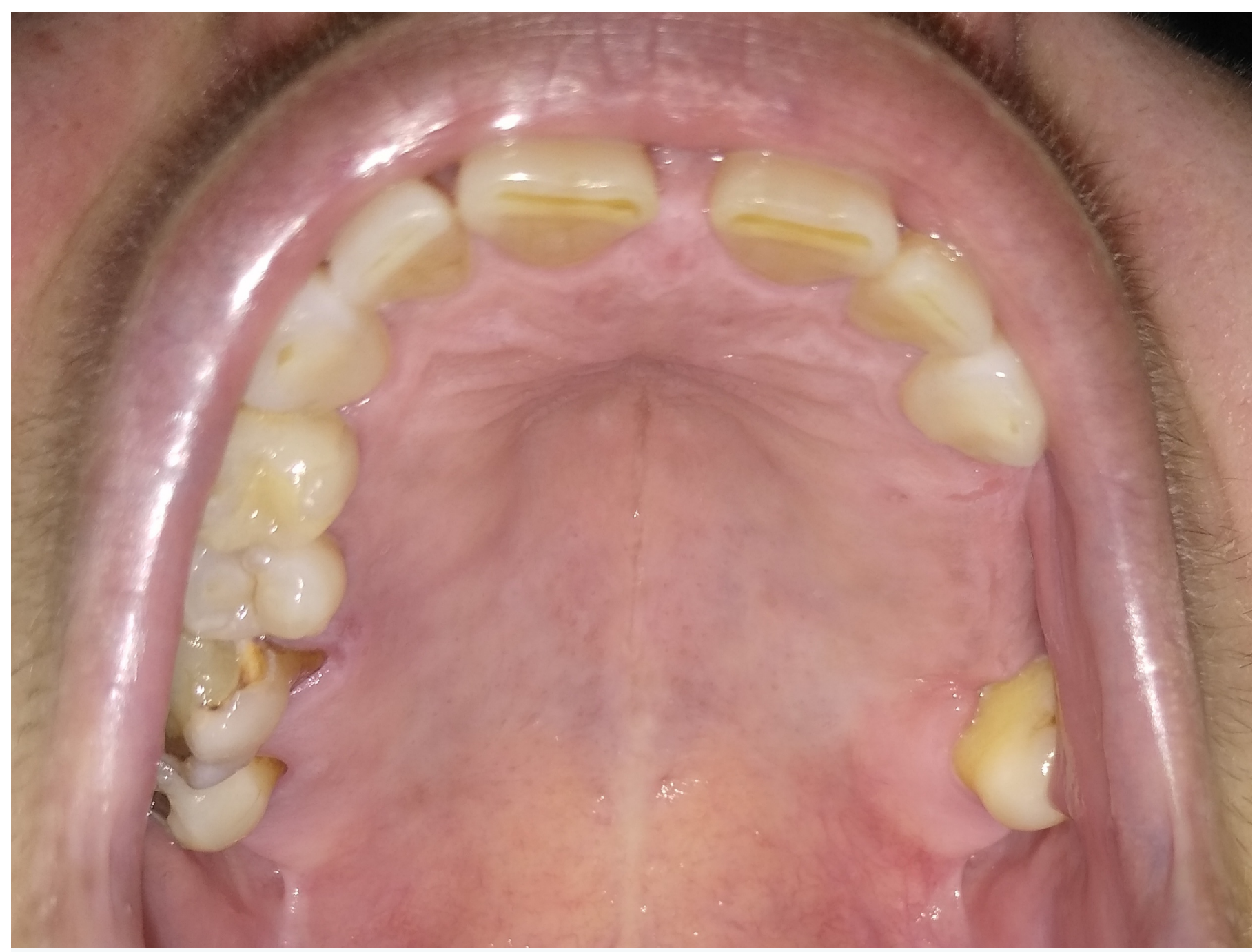

Fig. 5 Complete healing of the osteonecrosis after 10 days.

Results: At the 10-day follow-up, there was complete healing of the osteonecrosis (Fig. 5). Furthermore, no adverse effects were observed.

Conclusions. In conclusion, our case report showed how this particular alternative PDT could be considered a very useful for all refractory cases of MRONJ to conventional treatments.

\section{References}

1. Sarkarat F, Modarresi A, Chiniforush N, Yazdanparast L, Rakhshan V. Efficacy of Photodynamic Therapy in Minimizing Bisphosphonate-Related Osteonecrosis of the Jaws After Dental Extraction: A Preliminary Animal Study. J Oral Maxillofac Surg. 2019 Feb;77(2):307-314.

2. Ervolino E, Statkievicz C, Toro LF, de Mello-Neto JM, Cavazana TP, Issa JPM, Dornelles RCM, de Almeida JM, Nagata MJH, Okamoto R, Casatti CA, Garcia VG, Theodoro LH. Antimicrobial photodynamic therapy improves the alveolar repair process and prevents the occurrence of osteonecrosis of the jaws after tooth extraction in senile rats treated with zoledronate. Bone. 2019 Mar;120:101-113.

3. Magalhães IA, Forte CPF, Viana TSA, Teófilo CR, Lima Verde RMB, Magalhães DP, Praxedes Neto RAL, Lima RA, 
Dantas TS. Photobiomodulation and antimicrobial photodynamic therapy as adjunct in the treatment and prevention of osteoradionecrosis of the jaws: A case report. Photodiagnosis Photodyn Ther. 2020 Sep;31:101959.

4. Tartaroti NC, Marques MM, Naclério-Homem MDG, Migliorati CA, Zindel Deboni MC. Antimicrobial photodynamic and photobiomodulation adjuvant therapies for prevention and treatment of medication-related osteonecrosis of the jaws: Case series and long-term follow-up. Photodiagnosis Photodyn Ther. 2020 Mar;29:101651.

5. Poli PP, Souza FÁ, Maiorana C. Adjunctive use of antimicrobial photodynamic therapy in the treatment of medicationrelated osteonecrosis of the jaws: A case report. Photodiagnosis Photodyn Ther. 2018 Sep;23:99-101. 